\title{
Linx
}

Revue des linguistes de l'université Paris X Nanterre

$12 \mid 2002$

"Comme la lettre dit la vie »

\section{De semblance(s) en veraie semblance ; exemple d'un parcours du Merlin à la Queste}

\section{Nelly Andrieux-Reix et Emmanuèle Baumgartner}

\section{(2) OpenEdition}

\section{Journals}

Édition électronique

URL : http://journals.openedition.org/linx/1272

DOI : 10.4000/linx.1272

ISSN : 2118-9692

\section{Éditeur}

Presses universitaires de Paris Nanterre

\section{Édition imprimée}

Date de publication : 1 octobre 2002

Pagination : 13-29

ISSN : 0246-8743

\section{Référence électronique}

Nelly Andrieux-Reix et Emmanuèle Baumgartner, « De semblance(s) en veraie semblance ; exemple d'un parcours du Merlin à la Queste», Linx [En ligne], 12 | 2002, mis en ligne le 10 octobre 2012, consulté le 21 avril 2019. URL : http://journals.openedition.org/linx/1272 ; DOI : 10.4000/linx.1272

Ce document a été généré automatiquement le 21 avril 2019.

Département de Sciences du langage, Université Paris Ouest 


\title{
De semblance(s) en veraie semblance; exemple d'un parcours du Merlin à la Queste
}

\author{
Nelly Andrieux-Reix et Emmanuèle Baumgartner
}

Car la ou la verités vient avant, la figure doit estre arriere mise

(L'Estoire del saint Graal)

1 Une étrange veraie semblance fait irruption, au XIII siècle, dans la Queste del saint Graal, un récit dont elle fonde l'attente narrative et, en partie, la déception. Dénouant à sa façon l'énigme véhiculée depuis le Conte du Graal par le Graal auquel la Queste attache cette veraie semblance, elle se révèle et disparaît en même temps que la pseudo-relique. Or, on le sait, le XIII siècle est au plan littéraire le temps où se multiplie, d'un récit à l'autre, le questionnement sur un graal bientôt devenu le saint Graal et, au plan philosophique, le temps des interrogations et des débats sur les couleurs et les formes de la vérité. Semblant, semblance, comme toutes les unités du paradigme de sembl-, sont dans cette perspective des vocables-témoins dans la mesure où ils permettent de suivre les étapes d'une crise profonde dans les mentalités médiévales, de ce temps où s'est fragilisé le lien entre apparence et réalité, où est apparue la possibilité de manipuler sa propre apparence et, partant, de jeter le doute sur toutes les autres.

2 Etrange et peut-être unique, cette veraie semblance n'est rendue possible que par le statut lexical qu'a le substantif semblance dans la langue médiévale, où toutefois l'association avec veraie est encore tout à fait exceptionnelle. Dans la mesure où le terme semblance est exploité dans la Queste au-delà des possibilités habituelles du terme, en une transgression qui néanmoins les confirme, il nous a paru intéressant ${ }^{1} \mathrm{~d}^{\prime}$ examiner, en amont de la Queste essentiellement, le statut de semblance, mais aussi de semblant, pour y découvrir le mécanisme générateur de cette production lexicale éphémère. L'examen, au reste, s'est rapidement focalisé sur semblance et sur ses emplois dans les textes du Graal, en vers puis en prose, parce que, plus que d'autres ${ }^{2}$, ils ont exploité la valeur de ce terme, qu'ils en ont 
fait un élément clé de leur interrogation sur le sens à donner au Graal, cet objet incongru légué par Chrétien de Troyes.

\section{Sembl- dans le lexique médiéval}

3 La langue médiévale, toutes diversités textuelles confondues, accorde sensiblement les mêmes possibilités aux deux substantifs semblance et semblant, cette communauté relevant de leur appartenance au paradigme morphologique de la base sembl- dont ils sont tous deux dérivés. Dans l'aplatissement inévitable des données que fournit une collecte d'occurrences large mais non exhaustive pour chaque texte, les deux substantifs peuvent, en beaucoup de cas, apparaître comme variantes libres l'un de l'autre; mais ce nombre de cas diminue considérablement quand on examine le choix qu'opère chaque texte lorsque il se construit son vocabulaire: en semblant de se révèle, en pratique, beaucoup moins fréquent que en semblance de, de même que dire de sa semblance par rapport à dire de son semblant et que faire semblance de par rapport à faire semblant de, sans parler de plusieurs absences voire impossibilités de commutation qui s'observent entre les deux substantifs ${ }^{3}$. Les données des pratiques discursives médiévales mettent au jour, en effet, un partage entre ces deux vocables et l'observation de ce premier contraste servira de base à l'approche sémantique de semblance qui sera proposée ici.

Par sembl- il est signifié quelque chose qui se présente de façon manifeste, incontournable, majoritairement à la vue, mais sans exclusion d'autres perceptions sensorielles et de l'intellection : comme un donné à voir/percevoir offert à un récepteur qui, même s'il peut rester non explicite ou indéterminé, est toujours postulé par ce lexème et dont la présence est impliquée dans le schème actantiel qui lui est associé.

Cette orientation vers un récepteur/destinataire rend compte de l'ouverture, diversement manifestée dans l'ensemble du paradigme, à l'interprétation, l'évaluation, le jugement de ce récepteur. En même temps, caractéristique de sembl-, cette orientation peut opposer chacune des unités lexicales du paradigme à celles qui, relevant d'autres lexèmes, peuvent occasionnellement entrer avec elle en relation de synonymie partielle, à tout le moins de co-référence : c'est par là que sembler unipersonnel s'oppose à croire, penser, cuidier (mais, en revanche, se rapproche de estre avis), que semblance et semblant se distinguent de figure, forme, image. Et c'est ce qui explique, au sein du paradigme de sembl -, que les unités relevant de la même catégorie grammaticale puissent apparaître occasionnellement équivalentes lorsque la signification se limite à celle d'une apparence perçue, neutralisant ainsi d'autres différences, comme le montrent les exemples suivants :

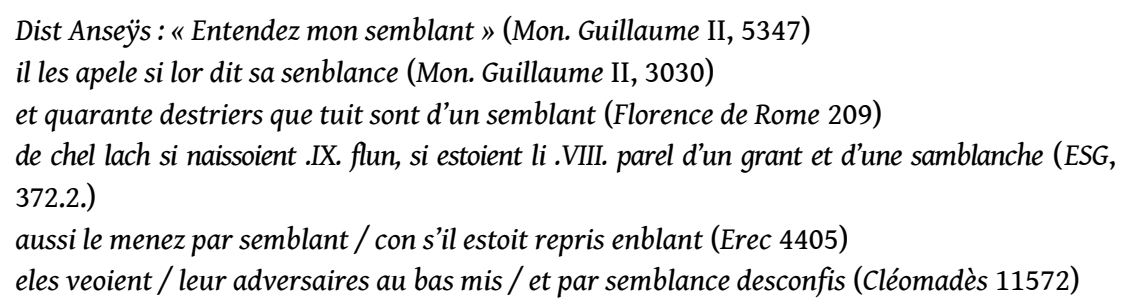

6 On notera également l'ambiguïté possible de par semblant, susceptible d'être interprété comme signifiant "à ce qu'il paraît» (à qqn qui en est le témoin) ou, quoique plus rarement, « aux apparences données » (par l'agent du procès), cette dernière traduction correspondant à toutes les occurrences de faire semblant : 
et li malades [un baron simulateur] s'esforce molt par samblant de parler (Merlin P 41. 62)

7 Mais si semblant et semblance peuvent commuter dans quelques formes syntaxiques, la donnée apparente qu'ils dénotent n'est pas nécessairement du même ordre selon chacun d'eux.

\subsection{Semblance et semblant. Des distributions informatives}

\subsubsection{Formes syntaxiques discriminantes.}

8 On a signalé plus haut la quasi-exclusivité d'emploi de semblance dans la locution en semblance de, celle de semblant dans les locutions faire/mostrer semblant ainsi que par nul semblant ${ }^{4}$, plus tard par tel semblant que :

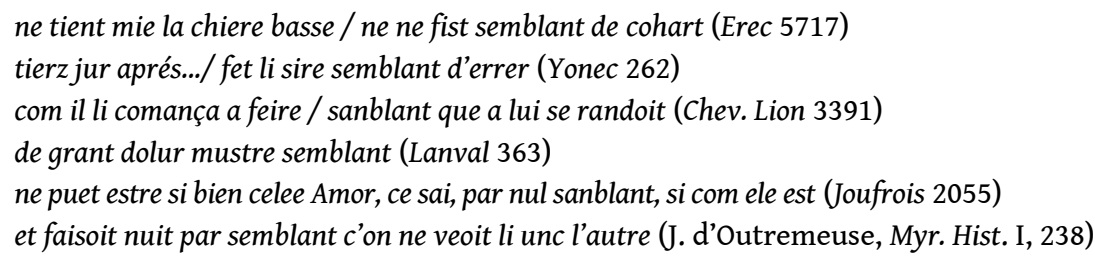

9 S'y ajoutent plusieurs situations syntaxiques admettant seulement l'un des deux substantifs, à l'exclusion de l'autre: seul semblance apparait possible comme objet déterminé d'un verbe ou d'une locution verbale dénotant un changement: prendre, se metre en, revenir en, muer, changier, doner..., ainsi que du verbe avoir :

Dïables esteit ki la gent / Deceveit par enchantement / Semblance de feme perneit / Par quei le pople deceveit (Brut 639)

Le cors, le vis, la cuntenance / e la parole e la semblance / que li cuens ad de Cornöaille / te ferai tut aveir senz faille (Brut 8708)

et maintenant qu'il s'en estoient lavé si cangoient tout leur samblanches et lor figures (ESG 93.21)

Et Merlin...prist la samblance dou garçon qui ot aportees les letres (Merlin P 37.57)

et si voil que vos sachiez que je ne muerai mais a piece ma semblance (Merlin P 39.59)

et prist congié de revenir en la semblance de quoi la gent de la terre le connoissoient (Merlin P 39.68/69)

En revanche, semblance apparaît exclu des expressions quantifiées contrairement à semblant qui peut ordinairement s'y trouver ${ }^{5}$ :

se il m'amast de fole amor / asez en veïsiez senblant (Béroul, Tristan 497)

a consoil vous diroie un pou de mon semblant (Florence de Rome 6309)

11 Même dans des situations syntaxiques identiques - où ils peuvent occasionnellement commuter - il ressort que chacun des deux termes est, en fait, rarement le substitut de l'autre.

䕕 Les adjectifs susceptibles de s'adjoindre à chacun de ces substantifs ne sont ni les mêmes ni dotés du même fonctionnement référentiel dans la qualification qu'ils apportent :

- bel, fier,...s'associent à semblant; par l'intermédiaire de semblant, l'adjectif peut qualifier l'être humain qui est le siège et la source de l'image ainsi dénotée: quelqu'un qui a un fier semblant est quelqu'un qui peut être dit lui-même fier il est de fier samblant (Mon. Guillaume I 518)

fiers par semblant come lions (Chevalier au lion 488)

par semblant ert cortois et sage (Hunbaut 3012) 
ou bien c'est la mine de quelqu'un ou encore son comportement qui sont qualifiés par l'intermédiaire de semblant (ce qui en explique la concurrence partielle avec chiere ${ }^{6}$ ):

por Aymeri biau semblant lor mostra (Aymeri de Narbonne 3874)

mes or oëz an quel meniere / a quel sanblant et a quel chiere / mes sire Yvains est herbergiez (Chev. au lion 5398)

encor vait cil solaz doblant / quant d'un ris ou d'un bel semblant / li membre ou d'une bele chiere / que fait li a s'amie chiere (Rose, 2662)

- vieil, sage, humain... qualifient semblance; ces adjectifs versent l'image dénotée par semblance dans une catégorie: la vieille semblance prise par Merlin n'est pas une vieille forme, mais l'apparence de quelqu'un de vieux ; l'umaine semblance dénomme même la catégorie définitoire de l'homme, non une apparence humaine ; à ce titre, ils fonctionnent plus comme des adjectifs relationnels

« en quel semblance estoiez vos quant vos parlastes a mon frere? - Je pris une vielle semblance saige » (Merlin P 35.24)

Et il [Merlin] ot prise une grant semblance vielle et de prodome (Merlin P 76. 24/25)

si les i firent metre [les lettres] par pitié d'umaine senblance li mesagier qui queroient Nascien (ESG 535.16)

Plusieurs de leurs cooccurrences consacrent cette opposition entre les deux substantifs :

tu les feis detraire por faire semblant que il t'avoit de sa mort pesé, mais ce ne fu mie bonne samblance des que tu pris le regne (Merlin P 30. 36/37) [Le devin reproche à Vertigier d'avoir simulé une souffrance qu'il n'éprouve pas]

et cele vit qu'il ot müee / la parole et la contenance / si parut bien a sa sanblance / qu'il ert iriez d'aucune chose / mes sanblant feire ne l'en ose (Conte du Graal 8046).

Lorsqu'il est constituant d'une locution verbale (employé avec faire/mostrer... ), semblant peut être qualifié de (molt) grant, de nul, révélant ainsi la possibilité de référer à du "gradable»:

mout faisoit grant semblant de mal pas afaitier (Aiol 6582)

que nul senblant de rien en face (Béroul, Tristan 2)

Même dans cet emploi (au reste rare) semblance n'apparaît pas susceptible de présenter ce trait.

17 㵧 Dans le cas d'une détermination en de par un élément nominal, les relations entre déterminé et déterminant peuvent être orientées différemment : ainsi le semblant de qqn/ qqch est-il toujours l'apparence produite par le déterminant, alors que si la semblance de qqn/qqch peut équivaloir à semblant, dans beaucoup d'autres cas il s'agira de l'apparence de ce qqn/qqch (le déterminant) prise/produite (temporairement) par qqn/qqch d'autre que ce déterminant :

semblant = semblance:

a l'entrer enz ont tuit löé/ Le bel semblant le roi Evrain (Erec 5559)

par ce qu'ele a oï parler de la semblance pense ele bien que ce soit Galaad (Queste, 10.23/24)

Soul de veoir sa contenance / sa grant beauté et sa semblance / a si les cuers de toz a lui (

Erec 5530)

semblant vs semblance:

Et quant il [le roi] s'en [de l'herbe] fu frotez, si ot tout apertement la semblance dou duc...Et Merlins revint arrieres a Ulfin si le transfigura en la semblance de Jordain...Et quant Ulfins vit le roi si se saingna et dist: «...coment poet estre nule semblance si bien faite d'un home a autre? (Merlin P 65. 7/8, 11/14)

18 㽞 Semblant peut référer à du «fragmentable» (ensemble fragmenté ou, plus tard, fragment d'un ensemble ${ }^{7}$ ), contrairement à semblance ${ }^{8}$ : 
et jo irai al Sarazin espan / si en vois vedeir alques de sun semblant (Ch. Roland 270)

e l'arcevesques lur dist de sun semblant (Ch.Roland, 1514)

a consoil vous diroie un pou de mon semblant (Florence de Rome 6309)

19 瀈 Semblant apparaît quasiment le seul ${ }^{9}$ à pouvoir signifier l'ensemble d'un comportement, la mine du visage voire l'accueil fait (dans des emplois proches de ceux de chiere) ou encore un indice de ceux-ci :

et se vos me faites bele chiere il ne m'en osseront ja faire samblant (Merlin P 39.58/59)

en son vis nul sanlant n'avoit / qu'il fust hom.. (Floire et Blancheflor 2638)

je n'iere mie si sourfaite que...que vous molt bonnement n'aiés / l'ostel et bon samblant a

nuit (Roman de la Violette,414)

las ! par losengier samblant / ne m'ont lessié de ma terre plain gant (Foucon de Candie 5966)

20 㵧 Certaines références enfin apparaissent propres à semblance :

- qu'on traduira par « comparaison»

Veez en par semblance / Dreite signifiance (Philippe de Thaon, Comput 2529/30

- ou par « apparition»

se li vint en avision / que devant lui en sa presance / s'aparissoit une samblance (Guillaume

de Palerne 1124)

- ou en référence à une appartenance catégorielle

ele [la bête extraordinaire] estoit diverse en toutes coses car ele avoit teste et col de brebis...et si avoit piés de chien et gambes et cuisses...et si avoit le pis et le cors et la crupe de woupil et la keue de lÿon : et si estoit la beste de diverses samblanches (ESG 19.15)

- voire à l'ensemble d'une catégorie

par pitié d'umaine semblance (ESG 535.16)

- semblance peut également référer à une représentation picturale (peinture, sculpture, dessin...) ou autrement symbolique :

Iluec a li leüns veü / Desur la piere une peinture / ... ... « Ki fist ceste semblance ici... ?» (Marie de France, Fables 73.13)

si poez conoistre en ceste espee la semblance des dous femes dont je vos ai oï parler car tot ausi come la Virge qui est a venir ...doit amender ce que nostre premiere mere meffist, tot autresi amendera cele pucele ce qe j'ai meffait en ceste espee (ESG 454.8)

21 De ces différences observables entre les deux substantifs il ressort que, avec ou sans verbe support comme faire/mostrer, semblant en français médiéval est toujours engagé dans la signification d'un procès : c'est un «nom processif ", qui dénote la production d'une apparence (plus proche en cela du verbe sembler dont il est originellement une forme). Ce caractère processif apparaît étranger à semblance, qui dénomme l'apparence elle-même, non la production de celle-ci, l'opposition de ce point de vue entre les deux étant d'ordre aspectal : semblance serait du côté de l'accompli, semblant de l'accomplissement (voir cidessus § 1.1.2., Merlin P 30.36).

Mais, qu'elle soit en cours de production ou déjà posée comme produite, l'image apparente elle-même n'est pas signifiée dans les deux vocables comme traitée de la même manière par le récepteur: avec semblant cette image se limite toujours au support délimité et défini par le déterminant; avec semblance l'image produite est toujours (ou peut toujours être) mise en relation avec une autre; ce qui explique à la fois la présence d'adjectifs relationnels et la quasi-exclusivité d'emploi dans en semblance de explicitant cette mise en relation; mais cela s'observe aussi là où semblance garde un statut syntaxique de substantif ${ }^{11}$, lorsqu'il est pourvu d'une détermination et/ou d'une qualification qui le fait référer à une réalité individuée (ex. la semblance de, sa semblance, une vieille semblance...). 
lance dénomme une apparence extérieure, mais qui peut glisser d'un support à l'autre parce qu'elle est toujours mise en adéquation avec une image déjà répertoriée et donc catégorisée : ex. la semblance dou garçon devenant celle de Merlin. Semblance est alors à rapprocher de forme, de figure mais ces derniers termes n'apparaissent pas engagés dans une relation de comparaison, d'adéquation (voir ci-après $\$ 1.2 .2$.)

\subsubsection{Premières conclusions.}

Semblant est ainsi engagé dans un procès d'extériorisation à partir d'une source qui reste le support comme la limite de son référent et à l'identification de laquelle il reste lié : $\mathrm{X}$ ne prend pas le semblant d'un autre. De ce fait, semblant ne dénomme pas nécessairement une forme, puisqu'il désigne avant tout une manifestation évidente d'un sujet dont cette manifestation peut être l'indice (fragmentaire) et la trace.

Tout autrement, semblance dénomme toujours une forme et une forme souvent associée à une transformation (une muance ou, autrement dit ailleurs, une de(s)-figuration ou transfiguration), susceptible de faire verser ce qui en est le siège dans une autre catégorie d'objets du monde :

Lors dist le rois a Ulfin : "Sez tu qui cist hom est qui ier parla a toi en la semblance d'un viel home? C'est cil meimes que tu as hui veu contrait " (Merlin P 62.42)

Et Ulfins demande: " Pourroit ce donc estre voirs que hom se pouist si desfigurer? Et que cist est qui einsis s'est desfigurez? (Merlin P 62.46)

Et Merlins revint arrieres a Ulfin si le transfigura en la semblance de Jordain... (Merlin P 65.11 sq).

En outre, et contrairement à semblant, semblance apparaît toujours engagé dans une sorte de comparaison par confrontation voire substitution d'images, comme le signale son association fréquente avec (tout) ausi come, (tout) autresi ; ce qui peut expliquer que la forme ainsi dénommée puisse parfois être floue, perçue globalement dans ses contours :

...mais en avant ne pooit il nule riens veoir del cors dont la mains mouvoit ne mais ke la samblanche seulement, qui estoit envolepee en la nue tout autresi com la samblanche d'un cors enseveli pert envolepee el suaire et si n'est pas li cors veüs apertement....tout en tel maniere veoit Nasciens le cors (ESG 377.21/22)

Ainsi s'explique que la semblance soit préférentiellement ${ }^{12} \mathrm{du}$ côté du masque, du couvert, de l'approximation, ce qui ne se confond pas nécessairement avec la non-vérité.

Contrairement à semblant, limité à la production d'une extériorité, éventuellement trompeuse, d'un sujet identifié, semblance dénote la re-production d'une image et selon des modes qui peuvent être différents, susceptibles de relever aussi bien de la ressemblance, "gradable " (à l'identique ou au plus ou moins rapprochant) que de la similitude, non "gradable », car fondée sur l'analogie et la sélection de traits saillants. C'est cette double possibilité13 qui, en français, fait occuper à semblance le champ de la similitudo latine, que d'ailleurs elle traduit ${ }^{14}$, et mieux que ne le fera similitude. La création, plus récente, de similitude ne paraît pas remonter au-delà du XIII ${ }^{e}$ siècle. ${ }^{15}$.

Entre ces deux modes de représentation et au sein des emplois de semblance il s'observe d'ailleurs une tendance au partage, correspondant partiellement (mais qui n'en est pas coextensif) à un partage d'ordre morphosyntaxique entre en semblance de et les autres emplois; partage qui peut aussi s'exprimer en termes de "nombrable» vs «nonnombrable». Et s'il est vrai que ces données distributionnelles ne suffisent point, elles permettent d'opérer de premières sélections dans toutes les possibilités de semblance. 


\subsection{Semblance et semblances}

Entre la semblance que Merlin prend du garçon qui a apporté un message et la relation établie ailleurs par en semblance de, par exemple à partir d'un morceau de substance à du pain ${ }^{16}$, il y a l'écart qui distingue l'identité d'une image (la ressemblance absolue) de la simple appartenance catégorielle qui admet la variation individuelle et, donc, la nonidentité de tous les traits.

\subsubsection{En semblance de ${ }^{17}$}

L'examen des éléments mis en regard par la locution adverbiale en semblance de qui s'insère entre eux révèle que ce sont souvent des vocables consignés dans la langue comme en relation d'hyperonymie (pour celui de gauche) à hyponymie (pour celui de droite) :

\begin{tabular}{|l|c|l|}
\hline un home & en semblance d(e) & prestre (Queste 99.13/14) \\
\hline un vieil home & - & home de religion (Queste 118.33-119.1) \\
\hline une maison & - & chapele (Queste 236.20) \\
\hline un vaissel & - & un hanap (ESG 116.6) \\
\hline une piece & - & pain (ESG 136.2, 137.3, 138.14) \\
\hline un rai de fu & - & espart (ESG 106.3) \\
\hline un engin & & - mail (ESG 283.7) \\
\hline
\end{tabular}

De gauche à droite, il s'opère, par la médiation de la locution, le passage d'une catégorie référentielle large à une plus étroite incluse dans la première: du premier groupe nominal au deuxième, l'image à percevoir du référent est affinée.

Mais les deux éléments peuvent aussi ne pas être en relation d'hyperonyme à hyponyme ni relever de la même catégorie super-ordonnée ${ }^{18}$ :

une table en semblance d(e)une autre table [qui l'a précédée et dont elle devient le symbole (Queste 74.32/3)]

\begin{tabular}{|l|c|l|}
\hline Notre Seigneur & en semblance & $d(e)$ cerf (ESG 801.8/14) \\
\hline \hline & - & enfant (ESG 281.213) \\
\hline & - & oisel (Queste 184.29) \\
\hline le fils de Dieu & - & Home (ESG 86.3) \\
\hline le sauveur & - & pain \\
\hline
\end{tabular}




\begin{tabular}{|l|c|l|}
\hline l'Eglyse- & en semblance d(e) & feme triste (Queste 185.17 sq) \\
\hline \hline l'enemi & - & ome de religion (Queste 186.5) \\
\hline- & - & cigne \\
\hline \hline fuisel & - & \\
\hline \hline une figure & - & emeraude (ESG 470.19) \\
\hline
\end{tabular}

Plus peut-être qu'à un changement de catégorie (une muance), en semblance de correspond alors à l'attribution d'une image définie à un référent qui n'en a pas ou ne peut en avoir.

Dans tous les cas, il s'opère ainsi une catégorisation dans l'ordre du sensible; et cette catégorisation, lorsqu'elle est signifiée par en semblance de, peut être engagée dans un sens à découvrir et avoir valeur de symbole ; ce qu'elle a très régulièrement dans les textes qui en font un emploi massif. Plus que de tous autres textes, c'est le cas des écritures du Graal, dont cela devient même une caractéristique.

De cette constance dans le fontionnement syntactico-référentiel de la locution se distinguent les emplois autres de semblance où, avec un statut de substantif, il réfère à une réalité discrète.

\subsubsection{Autres semblances}

Des exemples présentés ci-dessus (§ 1.1.3.) il ressort que, avec une même distribution syntaxique, les possibilités référentielles du substantif semblance sont très diverses : la semblance (tous les traits de l'aspect extérieur) du garçon, prise par Merlin, se distingue de la semblance (vision à interpréter) d'un songe autant que de la semblance (symbole) de deux femmes que peut être une épée; de cette dernière on rapprochera ce qui est l'une des plus anciennes, voire la plus ancienne attestation de semblance, dans le comput de Philippe de Thaon ${ }^{19}$ où le vocable dénomme une comparaison imagée :

\footnotetext{
La lune pert plus halte / une feie que altre / sulum le vëement / de cest' humaine gent; / mais nent est, ja n'ert, alte / plus une feiee que altre /.../ Veez en par semblance / Dreite signifiance: / se ci ert uns musters / Ki alques fust pleners / de lampes alumees / .../ sacez, qui en luin serait / e quis esguarderait, cele qui prof serait, / plus alte conceverait ; / l'altre qu'en lung serait / basse li semblerait... (Ph. Thaon, Comput 2529-2542)
}

Cela suffirait à prouver que, même si en semblance de peut paraître convenir de préférence pour tout transfert symbolique et pour l'engagement dans une senefiance, le partage entre les deux champs notionnels de la ressemblance et de la similitude (dont relève le symbole) ne s'opère pas par la seule situation morphosyntaxique; en fait, c'est la situation référentielle informée par le co-texte qui en décide.

semblant) qu'elle repose sur ce qu'il y a de commun aux deux processus de re-présentation (par ressemblance et par similitude), à savoir un transfert de traits sensibles, la différence entre les deux venant de ce que ce transfert peut affecter tous les traits d'une image déjà répertoriée aussi bien qu'en affecter seulement certains : 
- A la reproduction de tous les traits d'une image déjà existante correspondent la majorité des occurrences de semblance pourvu d'un déterminant, lui conférant l'univocité référentielle (la, ceste, sa).

- A la sélection de certains traits seulement correspondent généralement, mais non toujours, les occurrences de en semblance de ; ce mode de reproduction se divise luimême en deux, selon qu'il se fait avec ou sans transposition catégorielle :

* sans transposition, il y a seulement affectation à une sous-catégorie relevant de la même catégorie super-ordonnée ;

* avec transposition, selon un processus d'analogie, le transfert devient symbolique, ce dont est seul capable semblance (et majoritairement dans en semblance de) le terme se rapprochant en ce cas de signe, limité, lui, à la valeur symbolique.

Les premier et deuxième cas versent semblance au compte de la ressemblance (où il peut occasionnellement apparaître comme l'équivalent de semblant), le troisième le verse au compte de la similitude que, des deux substantifs, semblance est le seul à pouvoir signifier. Cette double possibilité contribue aussi à distinguer semblance de ses concurrents occasionnels, les principaux en étant image et figure, dans une moindre mesure forme, mais dont aucun n'inclut dans son sémantisme la relation avec le représenté, encore moins sa réception par un témoin susceptible de l'évaluer ${ }^{20}$ :

- dans image (qui, dans l'histoire, a remplacé semblance ${ }^{21}$ ) prédomine en ancien français le caractère à la fois matériel et iconique de la représentation, ce qui tend à l'exclure de la valeur symbolique (qu'a, en revanche, figure); en ancien français, image convient pour nommer toute représentation produite sur un support quelconque (peinture, dessin, sculpture...); avec cette référence, semblance serait une sorte d'image, mais perçue, en outre, comme faite en conformité avec une forme déjà existante :

...chele samblanche si estoit une ymage de fust a mervelle de grant biauté en guisse d'une feme.. (ESG 283.10)

lors fist...peindre dous dames qui traioient...un home......et fist l'ymage approprier au mielz qe ele onques pot a la forme d'Ypocras et les autres ymages as samblances des dames qui avoient ce fait (ESG 562.5)

- figure est aussi donné comme une sorte d'image :

Lors fist fere une grant figure d'or et haute, en figure d'ome semblable au plus que l'en pot en la forme d'Ypocras et apres refist faire une autre a la maniere de son neveu...desus cez douz ymages fist faire un arc volu (ESG 548.10/14)

mais ce que dénomme figure est généralement un type particulier de représentation, limité au contour, ne gardant du représenté que les traits essentiels ; ce qui rend apte figure à signifier le symbole, ces deux caractéristiques le distinguant d'image, mais le rapprochant de semblance:

ço mustre la peinture / si est dit par figure / la cüe par nature / mustre sainte escripture

(Ph. Thaon, Bestiaire 108)

tut a Deus averé / par la süe bunté / quant que sainte escripture / nus diseit par figure

(Ph. Thaon, Bestiaire 560) 22

41 La locution en figure de apparaît d'ailleurs comme l'équivalent, dans d'autres textes anciens, de en semblance $d e^{23}$ :

in figure de colomb volat a ciel (Eulalie 25) 
- la dénomination d'une forme, constante pour semblance, le fait entrer en coordination, aussi bien qu'en relation occasionnelle d'équivalence, avec forme :

cil qui...avoit forme et semblance de chien et $l i$ autre avoient forme de lyon; ...Li novismes qui venoit en guise de lyon et de flun (ESG 636.12)

lors fist...peindre dous dames qui traioient...un home......et fist l'ymage approprier au mielz qe ele onques pot a la forme d'Ypocras et les autres ymages as samblances des dames qui avoient ce fait (ESG 562.5)

Mais limité à la notion même de "forme ", soit d'un volume défini et comme découpé dans l'ensemble de son environnement, forme se distingue de semblance par l'absence de tous les autres traits qui caractérisent celui-ci, autant que le fait guise dans en guise de.

- la locution en guise de commute fréquemment avec en semblance de, mais guise est extérieur à la notion d'image, ne signifiant lui-même que la manière (comme a la maniere de) et la locution ne signifiant de ce fait que l'identité fonctionnelle - et occasionnelle - entre deux éléments :

Nostre Sires lor vint devant en guise d'oisel (Queste $184.6 / 7)$; cf. vos vint il mostrer en semblance d'oisel ( Queste, 184.29) li anemis li aparut en guise de fame (Queste 195.8) li Saint Esperiz descendi... en guise de feu (Queste 78.15)

aparoit il en guise de cerf blanc (Queste 236.9/10) chil huis...si tost com il clooit si chaoit par dedens un engiens de fier en guise de bare (ESG 283.2); cf. tantost caoit uns engiens de keuvre en samblanche d'un mail sour la premiere bare (ESG 283.7)

De cette double possibilité, le partage qui peut s'observer dans les occurrences de semblance est avant tout celui que se choisissent les textes, dont chacun, à partir de possibilités lexico-syntaxiques communes à tous ses contemporains, se construit un vocabulaire selon ses visées narratives. Quand celles-ci font de semblance l'un des attributs caractéristiques d'un actant/personnage ou, sur un autre plan, le constituant d'une dynamique narrative, le partage peut en venir à être radical : ainsi le Merlin en prose est-il presque tout entier du côté des semblances nombrables, interchangeables, jamais engagées dans une senefiance, tandis que, à l'autre extrémité24 d'une écriture, la Queste privilégie très majoritairement en semblance de, toujours engagé dans une senefiance; exploitation individuelle et parfois extrême ${ }^{25}$ des ressources d'une langue commune.

\section{Cheminements}

\subsection{Jeux de rimes: semblance/ senefiance}

L'une des plus anciennes sinon la plus ancienne des attestations de semblance se lit dans le Comput de Philippe de Thaon, sans doute composé vers 1113 ou 1119. Dans un long passage (en partie cité $\$ 1.2 .2$.), l'auteur s'attaque au problème du cours de la lune qui, aux yeux des ignorants, apparait plus ou moins « haute » selon les jours. Simple illusion d'optique - on pense au bâton de Pascal - dont il va démonter le mécanisme en utilisant une comparaison ainsi annoncée : veez en par semblance/dreite signifiance (vv.2529-30). La comparaison, la semblance convoquée, propose alors un détour qui fait appel au sens commun de l'auditoire en utilisant l'image de lampes régulièrement disposées dans une 
église. Pour qui les regarderait, la première semblerait la plus haute, les autres donnant progressivement l'impression (il s'agit bien entendu du trompe-l'œil de la "perspective ») de décroître régulièrement de taille. Ainsi du cours de la lune...Et la comparaison est ensuite redoublée par le recours au miroir et aux effets déformants qu'il peut susciter.

Dans cette première occurrence, semblance qualifie donc de manière abstraite - même si la démonstration du clerc utilise une image concrète - le procédé par lequel s'établit une analogie entre le monde connu et celui qui échappe à l'entendement humain; ou comment le recours à la semblance jette un pont entre l'un et l'autre, demostre, répèteront les romans du Graal, rend manifeste ce qui échappe à la saisie immanente et permet en ce cas de corriger l'erreur des sens. Au sein d'un univers mental fondé sur les correspondances entre l'univers sensible, domaine de la matière, et l'univers mystérieux/ divin qui l'englobe, la semblance apparaît ainsi comme l'outil idéal pour dépasser le sensible tout en y prenant un solide appui et approcher l'invisible ou l'incompréhensible. On sait comment les Bestiaires exploitent à fond ce système, mais à partir des natures, des traits physiques des animaux qu'ils sélectionnent pour leur aptitude à faire signe. On comprend donc aisément le rôle central qu'a pu jouer la semblance dans l'univers textuel co-fondé par le Graal et Merlin, un univers dont la ligne de force, à partir du Joseph de Robert de Boron, n'est autre que la quête, éventuellement la conquête non de l'objet Graal, mais du savoir absolu dont il est le dépositaire et qui permettra à l'élu de dépasser le monde des semblances pour atteindre à l'essence, à la connaissance de l'origine ; ce que ces textes désignent métaphoriquement par les « secrets du Graal ».

\section{2. Le grand jeu des semblances}

La distinction entre semblant et semblance s'observe déjà de manière significative dans la partie arthurienne du Brut de Wace. Lorsque Uter tombe amoureux d'Ygerne et la dévore des yeux, il est dit que :

Mult li ad ris e mult cluinié

E maint semblant fait d'amistié (vv. 43-44) ${ }^{26}$

Ces démonstrations d'amour correspondent, comme on le sait, à la vérité : Uter se meurt d'amour pour la duchesse de Cornouailles. Mais lorsqu'il s'agit d'inventer un stratagème pour approcher la dame, Merlin fait état auprès d'Uter de ses dons pour manipuler les semblances :

Figure d'ume sai muer

E l'un en l'altre tresturner.

L'un faz bien à l'altre sembler

E l'un faiz bien a l'altre per.

Le cors, le vis e la cuntenance,

E la parole et la semblance

Que li cuens ad de Cornoaille

Te ferai tut aveir senz faille (vv. 151-158)

Une herbe aux mystérieux pouvoirs lui permet en effet de donner à Uter la semblance, l'apparence physique du duc Garlois, à Ulfin le semblance de Jordan, lui-même déclarant qu'il prendra la semblance de Bretel (vv. 160-166). Au reste, cette semblance, dont le trio se débarrasse au matin en se débarbouillant le visage, semble plutôt relever de l'art du maquillage. Elle n'est en aucun cas assimilable à une métamorphose qui changerait l'essence des personnages. Elle s'attache tout au plus à modifier l'apparence extérieure et, 
dans ce cas, afin de tromper, la langue médiévale dit decevoir, l'adversaire. Corrobore cette valeur de semblance un exemple qui se lit au début ou presque du Brut. La statue de la «déesse» Diane qui révèlera en songe à Brutus son destin de conquérant de la Bretagne est ainsi présentée par Wace :

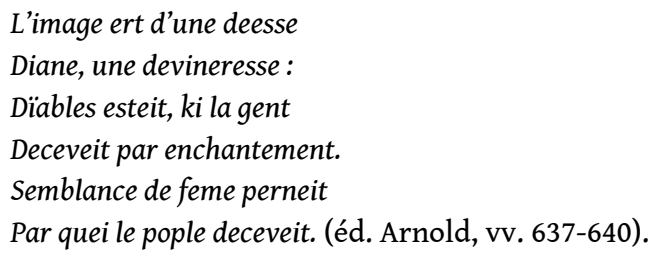

La semblance apparait donc dans ces deux exemples comme un moyen de tromper le monde, comme le support d'un trafic d'influence pour exercer un pouvoir abusif. Mais ne faut-il pas, dans le monde encore païen où vit Brutus, recourir à ce qui a alors cours, les prédictions des divinités païennes, quitte à récupérer ensuite cet héritage ambigu ? Et dans le cas d'Uter, n'est-il pas impératif que Merlin aide son protégé à posséder une femme « aimable », c'est-à-dire dont il puisse avoir un héritier ?

$\mathrm{Du}$ Brut au Merlin en prose, en revanche, le statut de semblance se modifie sensiblement. L'auteur anonyme du Merlin reprend sans doute à Wace le motif de la semblance par laquelle Uter abuse d'Ygerne et commet une sorte de viol semblable à celui qu'a commis le démon, "père » de Merlin. Mais les semblances que Merlin, l'être protéiforme par excellence, impose à son apparence corporelle et que multiplie le texte sont surtout des outils de travail et de pouvoir, au reste bien troubles. Il n'est nulle part précisé d'où Merlin tire cet art, qu'il faut sans doute imaginer diabolique. Dieu l'immuable a imposé à sa créature une semblance à son image. Déformer la semblance, en trafiquer, sont péchés graves. De ce point de vue Merlin risque le salut de son âme. Mais, dans le texte en prose, muer avec constance sa semblance est manifestement pour le devin un moyen d'en imposer à ses protégés, puis à Uter (voire, à l'occasion, de les divertir), afin d'accomplir les desseins de Dieu et, du même geste, sauver sans doute son âme ${ }^{27}$.

51 Dans le Merlin en prose d'autre part, les deux seules occurrences de la locution en semblance de se lisent dans l'entourage de semblance en fonction de substantif déterminé et désignant, comme dans le Brut, l'exacte reproduction des traits de trois hommes. On mettra ainsi en parallèle les emplois de semblance :

Et quant il [le roi] s'en [de l'herbe] fu frotez, si ot tout apertement la semblance dou duc $(65.7 / 8)$

Et Merlins revint arrieres a Ulfin si le transfigura en la semblance de Jordain...Et quant Ulfins vit le roi si se saingna et dist: "...coment poet estre nule semblance si bien faite d'un home a autre?» $(65.11 / 14)$

Quant il furent lavé si rorent lor semblance (66.29)

aux deux exemples suivants de en samblance de :

Et elle [Ygerne] li conte coment uns hom avoit geu a lui en semblance de son seingnor et avoit amené. II. homes en semblance de.II. homes que ses sires plus amoit (72.33/35)

Cette réduction jusqu'à l'effacement d'une tendance au partage qui s'observe ailleurs entre semblance(s) et en semblance de, est à notre avis significative d'un texte qui accorde une large prédominance à l'emploi substantif de ce vocable et qui l'affecte majoritairement à Merlin. Marque lexicale d'un des attributs majeurs conférés au fils du diable, semblance en dit les apparences diverses, interchangeables, formes - toutefois toujours humaines - sous lesquelles se présente successivement ${ }^{28}$, mais aussi se dérobe à la reconnaissance, un être par nature difficilement catégorisable et peut-être in-formel ; 
au point que se pose dans le Merlin comme dans La Suite du Roman de Merlin la question de la droite semblance de Merlin, question au reste soulevée par l'auteur du Merlin lui-même :

Et Merlins vint a la tente le roi en sa droite semblance que l'en le connoissoit (Merlin P 63.3) homme, d'un « homme sauvage ", d'un vieillard vêtu en clerc ou en magicien? L'auteur de la Suite $d u$ Merlin a perçu la difficulté puisque, après avoir fait apparaître sous de multiples semblances son héros à Arthur, très au fait, semble-t-il, des muances du devin,

"j'ai maintes fois oï dire que vous mués vostre samblance en tel couleur que vous volés et vostre forme en tel samblance qu'il vous plaist » (Suite du Merlin § 22)

il semble renoncer à ce jeu dans la grande scène de la reconnaissance d'Arthur et d'Ygerne. Alors que la reine exige que l'enchanteur lui dise son nom, Merlin

se met erraument en sa samblance vraie, en autele comme elle l'avoit autre fois veu [puis ajoute]: Dame, se vous ne savés mon nom, je le vous le dirai, mais je quidai que vous me conneussiés et vous le deussiés bien faire car autre fois me veistes vous ja. "Et ele le regarde, si le conoist maintenant (\$30).

Sans doute faut-il croire la reine et le commentaire du narrateur, mais quelle est à cet instant la semblance vraie de Merlin ${ }^{29}$ ? Aucune de ces semblances n'est d'autre part affectée d'une quelconque senefiance: Merlin en reste le seul maitre et elles n'ont d'autre fin que lui-même et que les facettes/facéties dont il joue, pour le plus grand bien, le cas échéant, du monde arthurien. C'est en effet avec l'irruption du Graal (le Merlin l'annonce mais il est alors occulté, hors champ de l'action) que se produit un changement radical et que les textes qui content son 'invention' et retracent son parcours vont faire de semblance un usage à la fois massif et, pour certains, très sélectif. Envoyées et maitrisées par Dieu luimême, les semblances feront désormais signe, engagées qu'elles seront dans un sens à découvrir. Il faudra au reste que Merlin s'efface devant le Graal, qu'il a pourtant annoncé, pour que se forge peu à peu le lien entre les semblances, toujours en mutation, et le point fixe (la ligne de fuite, mais en Dieu ) de la veraie semblance.

\section{Les écritures du Graal et la quête de la veraie semblance}

Le texte fondateur, en ce domaine comme en bien d'autres, est le Joseph en vers de Robert de Boron ${ }^{30}$. Un premier ensemble d'occurrences de semblance ${ }^{31}$ se lit dans l'épisode de la femme, Verrine (la future sainte Véronique), qui a conservé le linge où apparaît une semblance d'ome (v. 1507-8), la figure, le visage même du Christ, et qui porte remembrance de sa Passion ${ }^{32}$. Il est précisé que cette semblance s'est comme imprimée, 'imposée' sur le sydoine ${ }^{33}$, le linge qu'a utilisé Verrine pour éponger la sueur du visage du Christ :

Et quant en ma meison entrei

Et men sydoine regardei,

Ceste semblance y hei trouvee,

Tout ainsi comme ele est fourmee (Joseph, vv. 1611-1614)

Dans ce cas exceptionnel/miraculeux, l'image, la semblance, acquiert donc un statut tout à fait nouveau puisqu'elle est l'exact reflet, la véridique semblance, de la Sainte Face, le texte employant au reste visage (v. 1484) en alternance avec semblance pour caractériser l'image conservée par Véronique (à lire, rappelons-le, «vraie icône »...). De même que le Christ est à la fois homme et Dieu, de même sa semblance, image sensible aux sens, imprégnée dans la texture même du sydoine, signifie sans reste le mystère de son 
Incarnation, de sa présence réelle au monde des hommes et relie sans que subsiste la moindre faille l'univers terrestre et l'univers céleste ${ }^{34}$. La semblance est l'impression mêmedu visage même du Christ ${ }^{35}$.

A partir du Joseph, tout se passe comme si l'ambition des textes du Graal était d'inventer à partir de cette relique certifiée,

On l'apele la Veronique

C'on tient a Romme a grant relique (vv. 1747-8)

d'autres reliques de papier qui, toutefois, ne pourraient qu'en être le simulacre - la semblance - dans l'univers de la fiction. Robert de Boron en effet ne franchit pas les bornes : en aucun autre passage n'apparaissent de semblances qui aient l'absolue réalité de la Véronique. Lorsque le Christ, visitant Joseph, établit une série de concordances entre son sépulcre et l'autel, son suaire et le corporal, le vaissel où Joseph a recueilli son sang (qui n'est pas encore qualifié de graal), et le calice, la pierre du sépulcre et la patène, il emploie systématiquement le verbe "être » au futur qui pose sans reste l'équivalence entre les deux séries et qui redéploie l'équivalence fondatrice entre le pain et le vin et le corps et le sang du Christ ${ }^{36}$ instituée lors de la Cène. Mais le Graal, nom autre que la fiction donne peu après, par l'intermédiaire d'un certain Petrus, au vaissel de Joseph d'Arimathie, nom imposé par l'homme et non par le Christ, en reste, lui, au stade de la semblance, de l'objet témoin, qui fait signe vers le sacré, mais qui n'est pas le signe ${ }^{37}$, comme l'est sans équivoque possible la Sainte Véronique (ou le Saint Suaire). De manière significative d'ailleurs, c'est alors le couple demostrance en rime avec senefiance qui apparait dans le texte : le parcours ternaire du Graal, de Joseph au troisième et dernier gardien de la 'relique', aura pour fonction de manifester et de signifier dans le monde de la fiction le mystère de la Trinité :

Lors sera la senefiance

Acomplie et la demoustrance

De la benoite Trinité

Qu'avons en trois parz devisé (vv. 3371-4).

A partir du Joseph, se construit ainsi autour et à partir du Graal un univers de semblances, en général fabriquées par Dieu - mais le diable tentera parfois de faire des contre-façons et dotées d'une senefiance cryptée qu'il importe de décrypter afin de dissiper l'obscurité des mystères divins, d'en pénétrer les secrets ; car, comme le rappelle le Christ dans le Joseph, et la formulation de la prose est plus explicite encore : quant chose est aperte, n'i a mestier senefiance (1. 319)... Déclaration en laquelle on peut voir la source de l'immense labeur qu'accompliront les écrivains du Graal au XIII ${ }^{e}$ siècle pour encoder/ crypter des demostrances, reprises pour l'essentiel à la fiction arthurienne, dont ils se feront un plaisir et une spécialité de décoder (à peu de frais) la senefiance ...Mais déjà « je » Robert de Boron prévenait ses lecteurs : «si je renonce à conter les branches qui composent l'Estoire dou Graal, elles tomberont dans l'oubli et nul ne saura en quel senefiance / j'en aroie feit dessevrance » (3513-4); ce que l'on pourrait très lourdement gloser par : personne ne saura comment j'aurais réparti, disposé ces branches, pour en faire un ensemble signifiant ${ }^{38}$.

Nous n'avons aucun moyen de savoir si c'est Robert de Boron ou des écrivains qui en ont habilement usurpé le nom et l'autorité qui ont mené à bien la trilogie en prose Jospeh, Merlin, Perceval (dit aussi Didot-Perceval),mais les prosateurs qui, au XIII ${ }^{\mathrm{e}}$ siècle, ont retravaillé le motif du Graal et de sa quête se sont évertués à construire à partir du référent arthurien un monde signifiant dont les semblances, les manifestations ou autres demostrances, forment désormais l'armature ${ }^{39}$. Manière sans doute de substituer à l'agressif arbitraire de l'aventure chevaleresque, trame ordinaire des récits arthuriens en 
vers, un univers où l'aventure fait et doit faire sens, et dont la manifestation la plus aboutie, la plus systématique, est la Queste (ou mieux Les Aventures) del saint Graal.

Discrètement mise en place dans la partie du Lancelot où s'annonce le retour du Graal et où se multiplient les visites des chevaliers - Gauvain, Lancelot, Bohort -, au château de Corbenic, résidence de la relique ${ }^{40}$, la machine à produire des semblances tourne à plein régime dans la Queste del saint Graal. Au prix d'un changement essentiel : dans le Lancelot et dans la Queste ainsi que dans l'énigmatique Perlesvaus, le Graal et la Lance qui saigne ne sont plus des objets dont il faut retracer l'origine et que doit patiemment quêter l'Elu, celui qui posera les bonnes questions au Roi Pêcheur. Ce sont les différentes Continuations du Conte du Graal (en vers) dont le héros reste Perceval, qui se sont placées sur ce terrain, préparé par Chrétien. Dans le Perlesvaus et dans la Queste, le Graal devient tout spécialement la manifestation de Dieu et de la grâce divine dans l'univers arthurien dans lequel il se met en aventure. Le Graal multiplie les demostrances en revêtant des semblances diverses dont il importe dans un premier temps de décoder la senefiance, mais au trio d'élus de la Queste est tout spécialement réservé le droit de passer du simple décodage des semblances à la vision progressive (totale pour Galaad) des secrets du Graal, de sa veraie semblance.

61 L'enjeu se précise peu à peu, du Perlesvaus à la Queste. Le Perlesvaus semble plutôt confronté à la déconcertante mobilité des semblances du Graal et du monde. Comme le déclare l'auteur dans un passage souvent cité :

Josephes nos tesmoigne que les semblances des isles se muoient por les diverses aventures qui par le plaisir de Deu i avenoient (p. 282)

pour la plus grande joie des chevaliers qui trouvent toujours de nouvelles aventures à mener pour établir sur terre la « Novele Loi » (p. 282).

Mais il se pourrait aussi bien que l'objet même du Perlesvaus soit de donner forme textuelle à l'anamorphose généralisée, au combat toujours renouvelé contre les forces du Mal. Toutes les semblances, même les plus inattendues ou les plus monstrueuses, sont alors bonnes à prendre, car elles manifestent dans leur diversité, dans leurs muances ${ }^{41}$, l'infinie fragmentation du monde, le brouillage et la confusion qui y règnent depuis que l'homme est tombé dans la région de la «dissemblance». Toutefois, les semblances du Graal sont déjà dans le Perlesvaus un moyen pour les élus d'accéder à la vision directe des grands mystères de la Foi, mystère de l'Incarnation, mystère de l'Eucharistie, mystère de la Trinité, de cette anamorphose éternisée par laquelle le 3 se fait 1 pour redevenir $3^{42}$.

On peut longuement épiloguer sur la chronologie relative de la Queste et du Perlesvaus. Leur rapport aux semblances reste de toute manière dissemblable. Alors que dans le Perlesvaus, le Graal et ses muances se manifestent spontanément, pourrait-on dire, le but âprement recherché par les chevaliers de la Queste est de voir apertement un Graal qui est apparu « couvert » d'un samit blanc à la cour d'Arthur et qui va ensuite se manifester sous diverses semblances, qui sont autant d'approches dans la quête de la veraie semblance. Les nombreuses semblances du Graal offertes aux quêteurs - la formule en semblance de recouvre dans la Queste $60 \%$ des occurrences du terme - renvoient toutes à des senefiances , qu'il importe de décoder et qui forment pour l'essentiel l'enseignement moral et religieux dispensé par la voix autorisée des ermites. Mais c'est le passage, le saut métaphysique de la semblance à la veraie semblance, à la vision sans voile du Graal ou plus précisément de son contenu, qui donne au récit sa dynamique d'ensemble. Comme le déclare Gauvain au début du récit, en jurant le premier d'entrer en quête du Graal qui vient de se manifester à la cour d'Arthur : 
De tant sont il [les compagnons de la Table ronde et futurs quêteurs] engignié qu'il nel porent veoir apertement ançois lor en fu coverte la vraie semblance...; por coi je endroit moi faz orendroit un veu, que le matin, sanz plus atendre enterrai en la queste...ne ne revendrai a cort...devant que je l'aie veu plus apertement qu'il ne m'a ci esté demostrez (16, 1. 17-23). du Graal qui lui est réservée à Corbenic :

...Je voi que vos tenez mon Sauveor et ma redemption en semblance de pain; et en tel maniere nel veisse je pas ne mi oil, qui sont si terrien qu'il ne pueent veoir les esperitex choses nel me lessent autrement veoir, ainz m'en tolent la veraie semblance, car je ne dout je mie que ce ne soit veraie char et verais hons et enterine deïté (167, 1. 4-9).

C'est en effet au seul Galaad qu'est réservée au terme de la Queste la vision parfaite qui comble totalement son désir mais qui reste finalement indicible :

Et il se tret avant et regarde dedenz le saint Vessel. Et si tost come il i ot regardé si comence a trembler molt durement si tost come la mortel char commença a regarder les esperitex choses. Lors tent Galaad ses meins vers le ciel et dit : "Sire, toi ador ge et merci de ce que tu m'as acompli mon desirrier car ore voi ge tot apertement ce que langue ne porroit descrire ne cuer penser. Ici voi ge la començaille des granz hardemenz et l'achoison des proeces; ici voi ge les merveilles de totes autres merveilles !... (277.32 - 278.7).

En rupture avec le Conte du Graal et ses Continuations, le Graal est devenu dans la Queste le lieu d'un regard assez pur, assez nettoyé des ordures du monde de la matière, pour traverser et dissoudre l'enveloppe matérielle sous laquelle se dissimulent ses « secrets». Pour se demostrer, pour attirer les quêteurs, le Graal a cependant recours à diverses semblances, représentations approximatives, à la mesure des pécheurs à qui elles sont destinées, mais qui permettent peu à peu de se rapprocher de la veraie semblance.

Le texte lui-même est d'emblée fondé sur le concept de ressemblance, au sens physique déjà: dans l'ouverture du récit est systématiquement rappelée la ressemblance de Lancelot et de Galaad, même si le lecteur sait bien que Galaad aura par rapport à son père un « surplus » de valeur et de connivence avec le sacré. L'auteur transpose ensuite cette notion au plan théologique en reprenant à l'exégèse médiévale la technique de la mise en concordance. Mais les ressemblances s'établissent alors non entre l'Ancien et le Nouveau Testament, mais entre le monde arthurien (référent "concret» du récit) et d'autres mondes: Ancien Testament, Nouveau Testament, temps terrestre du Christ, temps de Josephé. L'expression clé est la formule très fréquente tout aussi com qui établit le lien entre deux espaces-temps, deux situations, etc. ${ }^{43}$ Dans la majorité des cas, la relation ainsi (arbitrairement) posée se matérialise dans la forme d'une semblance dont la fonction est double. Concrète, c'est-à-dire incarnée dans le monde arthurien, elle rend compte, le plus souvent sans équivoque ${ }^{44}$, du concept ou de l'événement comparé. On l'a vu, le feu, le pain, le cerf blanc sont semblances de Dieu (p. 78, 112), un prêtre est samblance du Christ (p. 99), un évêque semblance de Josephé (p. 277,278), une toaille, samblance de l'étoffe vermeille qui recouvre le Graal (p. 273), un enfant est semblance de l'Incarnation du Christ (p. 269), une femme affligée est semblance de l'Eglise (p.185), un cygne est semblance du diable, mais un noir oisel semblance de Dieu (p. 185) ; et Bohort en ce cas perd un moment ses repères...

68 Toutes ces semblances très prévisibles sont du même geste porteuses d'une senefiance, d'une mise en concordance avec d'autres temps, d'autres événements, d'autres comportements que se chargent avec zèle d'expliciter les ermites en poussant jusqu'à la caricature les procédés de l'exégèse. Mais dans la Queste, la semblance, manifestation à la fois similaire et simulée, ne recouvre jamais complètement ce qu'elle a à charge de 
montrer. Un crucifix porte sur lui le signe de la Veraie Croiz, la figure du Crucifié, il n'est pas la Veraie Croiz (p. 65). La semblance est un substitut toujours imparfait, et parfois impertinent puisque le diable peut pervertir les signes. Le travail de l'écrivain consiste alors à établir une hiérarchie entre les semblances qui recoupe bien entendu la hiérarchie des quêteurs, de Galaad l'élu au couple condamné Gauvain- Lionel. Au sommet de la hiérarchie se lit l'adjectif verai qui qualifie, ailleurs dans le texte, l'être, la chose, le concept qui ne participent pas seulement de l'essence mais qui sont l'essence, l'être même. On relève ainsi dans la Queste des formules du type la veraie Croiz (p. 32), le verai Crucifié ( p. 166), le verai Peres ( p. 215), les veraies noveles, (p. 79 et 231), la veraie lumiere (le Christ, p. 114,123), la veraie confession (p. 128, 131, 162), le verai cors Jesus Christ (p. 252), la veraie estoire (la Bible ou la fiction du Graal ? p. 214), le verai chevalier (Galaad, p. 116, 117, 159, 249).

Dans la Queste, la fonction médiatrice de la semblance est ainsi d'attirer les meilleurs, Lancelot, Bohort, Perceval, Galaad, vers la vision directe, celle de la veraie semblance, d'en inscrire le désir dans leurs cœurs, d'en hiérarchiser bien entendu l'accès. Cette vision est d'abord troublée, floue. Lorsque Lancelot assiste à la liturgie du Graal qui lui est réservée, il voit nettement les objets, les anges, l'officiant. Mais voit-il en direct ou croit-il voir (il fu avis a Lancelot, indique le texte) cette étrange scène qui unit le mystère de la Trinité à celui de l'Eucharistie (de la Présence réelle du corps du Christ dans l'hostie)?

Et quant il [l'officiant] dut lever corpus domini, il fu avis a Lancelot que desus les mains au preudome en haut avoit trois homes, dont li dui metoient le plus juene entre les mains au provoire; et il le levoit en haut, si fesoit semblant qu'il le mostrast au pueple (p. 255, 1. 21-25)

Plus complexe encore est le jeu des semblances et des semblants dans la liturgie (p. 268-9) dont Joséphé en samblance d'evesque est l'officiant. Les chevaliers présents voient tous les objets nécessaires au rituel : table d'argent, anges, cierges, touaille de vermeil samit, lance qui saigne et dont le sang s'écoule dans le vase du Graal, que Joséphé recouvre de la toaille. Ce dernier geste a son importance puisque, au moment où fist Josephes semblant que il entrast ou sacrement de la messe, les semblances alors énumérées, l'hostie faite en semblance de pain qu'élève l'officiant et dans laquelle pénètre une figure en samblance d'enfant, se muent, semble-t-il, en vision parfaite, les assistants voyant alors apertement que le pain a (et non semble avoir) forme d'ome charnel (p. 269), alors même que reste encore différé le regard qui plongerait au cœur même du veissel.

71 Ainsi se déroule jusqu'à la fin du récit le scénario qui tente, d'une semblance à l'autre, de cerner le mystère. Il va de soi cependant que la vision de l'intérieur même du saint Vase, $\mathrm{du}$ creuset où l'esprit informe la matière (où s'opère/se voit le mystère de l'origine) est réservé au seul Galaad qui emporte aussitôt dans l'au-delà le secret indicible et impossible à manifester de la veraie semblance.

72 Les personnages de la Queste et ses lecteurs ne cheminent donc pas dans un univers traversé de demostrances insolites, comme l'est celui du Perlesvaus. Les demostrances du Graal se manifestent par le support d'une semblance qui met en liaison le céleste et le terrestre et dont il convient ensuite de chercher la senefiance. Au delà de la visée moralisante - mais quel lecteur s'est jamais sanctifié à la lecture de la Queste? - l'enjeu est de taille. Il est autant d'unir par le langage l'au-delà et l'ici-bas, de rendre Dieu sensible aux sens, que de montrer comment l'écrivain peut, à partir d'un univers fictionnel, d'une relique de papier, disposer à son gré les signes, établir comme il l'entend les semblances, reconstituer les fragments du divin à la semblance d'un monde arthurien lui-même 
engendré, y compris Galaad et le Graal, par les pouvoirs de la fiction. Réservée à Galaad, la vision de la veraie semblance reste sans doute indicible donc hors texte. Elle témoigne du moins du désir fou qui incite l'écrivain profane à dépasser l'univers des semblances, à comprendre le mystère, à passer, un jour peut-être, de la rhétorique laborieuse des comparaisons, du « tout aussi com ", à une poétique de la métaphore.

A la faveur d'un parcours narratif propre à la Queste et dont la dynamique a eu une incidence inattendue sur les possibilités sémantiques d'un vocable, toutes les occurrences multipliées de semblance tendraient ainsi vers l'abolition de l'altérité qui pourtant fonde l'emploi du vocable ; comme si en cette ultime et unique veraie semblance se rejoignaient, selon une géométrie non euclidienne, les images parallèles et pourtant indissociables de ce que signifie semblance, où coïncideraient en une fusion unique et fulgurante apparence et essence.

Position parousique et insoutenable pour un vocable, qui, d'ailleurs, n'a pas été soutenue : après cette implosion référentielle, la veraie semblance disparaîtra de la langue, tout comme, après la vision aussi insoutenable de Galaad, le Graal disparaitra dans le horschamp du texte arthurien.

\section{La desloial semblance de l'Estoire del saint Graal}

On admet généralement que l'Estoire del saint Graal est postérieure à la Queste bien que ce texte ait été visiblement composé pour introduire au cycle du Lancelot-Graal ${ }^{45}$. Quoi qu'il en soit, les semblances y jouent également un rôle important, mais elles sont d'emblée subordonnées à la vision, moyen qu'utilise de préférence la divinité pour révéler ses mystères aux élus. C'est en effet la vision du mystère de la Trinité à laquelle accède dans le prologue le clerc ravi en esprit par le Christ (le corps est, comme dans la Queste, obstacle essentiel à la vision des mystères) qui informe le récit, lui-même transcription du « livret» que le Christ a donné à son futur scripteur. Dans le sillage de cette vision inaugurale et fondatrice, tout au long du récit, les visions et songes accordés aux personnages l'emportent de loin, en quantité, en qualité et en effets (la conversion dans la plupart des cas) sur les semblances. On relira de ce point de vue le déploiement des visions totales réservées à Joséphé au seuil de l'arche et à Joseph (\$111 et ss. et 115) et que, dit le texte, tous deux veoient tout apiertement $(118,1.22)$. Plus précisément encore, les semblances en ce cas ne sont pas des approximations, mais imagent sans reste les différents mystères. Ainsi par exemple de l'apparition du Christ dans laquelle se superposent la semblance du Christ au matin de sa Résurrection, celle, identique, sous laquelle il est apparu à Joseph (jeté en prison par les Juifs) et la semblance légèrement modifiée du Dieu vêtu comme un prêtre; précision qui annonce la consécration de Joséphé comme premier évêque par le Christ lui-même, la nouvelle semblance engageant ainsi le récit dans le devenir du temps chrétien

Après esgardoit Joseph, si veoit issir hors Jhesucrist en autrestel samblanche com il aparut en la chartre ou il estoit en prison, quant il fu issus del sepulcre et en cors et en esperit au jour de sa resurrection. En cheste samblanche le vit Joseph venir hors, fors tant seulement ke il avoit ore vestus tous les vestemens ke prestres doit vestir quant il veut faire le sacrement Nostre Signeur (\$118, 1. 13-18).

Il est impossible d'étudier ici dans le détail de ses nombreuses semblances le texte très dense de l'ESG. On se contentera donc de quelques indications. La beste de diverses semblances qui apparaît à «l'auteur » parti en quête du « livret » donné par le Christ (§ 
$19,1.15)$, est à bien des égards emblématique d'un récit lui aussi très "divers", qui multiplie les lieux de l'aventure et de l'initiation mystique, les îles notamment ${ }^{46}$, ainsi que les personnages destinés à fonder les lignées qui peupleront le monde arthurien, et qui accueille des récits dans le récit aux tonalités les plus disparates. Sur le modèle de la Queste, l'auteur recourt lui aussi avec fréquence aux semblances 'ordinaires' qui engagent des senefiances le plus souvent transparentes. On retrouve ainsi le Saint-Esprit en samblanche d'espars (\$106), une pieche en samblanche de pain mais qui, lorsque Joséphé l'approche de sa bouche, devient un cors tous entiers ( 136$)$, un diable en samblanche d'un home (\$252) ou encore, dans la vision accordée à Nascien (vision déjà donnée par la Queste ), les samblances, elles-mêmes déjà symboliques, de ses descendants: les futurs bons serviteurs de la foi apparaissent en semblance de lion (\$641), le huitième (Lancelot) en forme ou en samblanche de chien (\$642) et le neuvième (Galaad) en guise de lyon et de flun (\$636).

77 Curieusement cependant, la semblance qui dans la plupart des cas oriente l'homme vers Dieu et ses mystères, tend parfois dans l'ESG à glisser du côté du simulacre impie et à devenir une desloial semblance. A plusieurs reprises en effet, il est fait mention de semblances si parfaites - ce sont alors des statues ou des peintures - qu'elles incitent les hommes à l'idolâtrie ou au fétichisme. Ainsi dans l'ESG, christianiser un peuple et son roi, c'est bien souvent détruire les idoles qu'ils adorent et auxquelles ils prêtent des pouvoirs exorbitants. On retrouve alors un grand classique des chansons de geste, la scène du bris des idoles, de la destruction du quatuor Mahomet, Jupiter, Tervagan, Apollin (\$ 782). Mais lorsque Joseph met en doute le pouvoir réel de ces « images » sculptées par l'homme, son interlocuteur sarrasin argumente de façon intéressante sur le pouvoir à accorder, ou plutôt à ne pas accorder, aux ymages faites à la semblance des dieux païens :

Voirement di ge qe li ymage ont puissance, non pas par els, mais par cels de cui il ont semblance, ce est par les dex en cui semblance il sunt fait et en cui henor nos les cultivons. Ce sai ge bien que li ymage tant seulement d'els meesmes ne poent aidier, mais chascuns puet par la grace de celui en qui forme il est fez (\$ 783 ).

Bien entendu, Joseph accomplira le miracle qui convertira le sarrasin, mais le lecteur peut tout aussi bien mettre en question l'efficacité et la valeur réelle des semblances en général et s'interroger sur les frontières entre l'idole païenne et l'icône ou l'image sainte. D'autant plus que d'autres passages réaffirment le pouvoir monstrueux que peuvent exercer sur les hommes des semblances trop ressemblantes. Ainsi de l'épisode consacré à Hippocrate et à ses déboires amoureux ${ }^{47}$. La dame dont il s'est épris et qui veut ridiculiser le filosophe dont elle a mis en doute les pouvoirs guérisseurs, fait peindre

en une table de fust [sur un chevalet ?] dous dames qui traioient contremont une tor un home en un vaissel de fust et fist l'ymage approprier au mielz qe ele onges pot a la forme d'Ypocras et les autres ymages as semblances des dames qui ce avoient fait ( $\$ 562$ ).

Or, la conformité des images avec la mise en scène qui a couvert de ridicule Hippocrate est si parfaite que la dame peut affirmer à l'empereur que la vérité ne se trouve pas du côté du philosophe - il n'a pas pu ressusciter comme il l'a prétendu un homme mort mais que en ces ymages qui sunt en ceste table painz n'a se verité non ( $(563)$.

Et Hippocrate est bien forcé d'en convenir.

81 Le pouvoir des semblances à faire sens par elles-mêmes se fait plus pervers et bien plus dangereux encore dans l'épisode au cours duquel Joséphé démasque les pratiques diaboliques du roi Mordrain qui est encore païen à ce stade du récit ${ }^{48}$. Mordrain garde en effet dans un souterrain secret 
une desloial samblanche ${ }^{49}$...si estoit une ymage de fust a merveille de grant biauté en guisse de feme et si estoit vestue de si riches reubes com li rois les pooit trover plus riches et plus precieuses. A chele ymage gisoit li rois carnelment et de si grant amour l'avoit amee bien .XV. ans ke nus hom ne peüst avoir grignour amour a nule feme mortel (§ 282).

Pratiquer simultanément fétichisme et onanisme est bien sûr inadmissible. A Dieu d'agir et de donner enfin au roi le courage d'exhiber au grand jour sa poupée de bois et de la jeter dans le feu avec la reube ke ele avoit vestue et li fust dedens (\$283).

Christianiser le monde, ne serait-ce pas alors en finir avec les semblances pour que seul règne un Dieu invisible? L' ESG, dernier texte à prendre sérieusement pour thème le Graal, jette ainsi le doute sur la valeur trouble des semblances, trop ancrées dans le monde sensible et trop susceptibles de concentrer sur elles et sur elles seules le regard/le désir de l'homme. Seules les visions mystiques, émanant directement du Ciel, permettent désormais à l'élu d'entrer au royaume de la veraie semblance. A moins que l'auteur anonyme de l'ESG, faisant coexister vraies et perverses semblances, n'attire ainsi l'attention sur le récit en semblance d'Evangile qu'il a imaginé, sur cette très desloial semblance qui inscrirait définitivement les fondations du monde arthurien, 'l'invention' $\mathrm{du}$ Graal et son parcours sous le signe de la fiction...

Quelles qu'aient pu être au XIII ${ }^{\mathrm{e}}$ siècle - ou ne pas être ${ }^{50}$ - les relations directes entre débats de théologiens et préoccupations de romanciers, il est impossible que la Queste et l'Estoire del saint Graal aient échappé à la mentalité iconoclaste des Cisterciens et, parallèlement, à la recherche de la vérité au-delà des apparences. En relèvent de toute évidence, la création, au fil du discours de la Queste, d'une veraie semblance unique - autant qu'on puisse en connaître - pour annoncer l'indicible face à face avec le Graal ${ }^{51}$ et, dans l' ESG, la dévalorisation de la semblance, lors même qu'elle n'est pas explicitement qualifiée de desloial. Toutefois, alors que le Graal disparait à tout jamais après cette vision singulière de la Queste ${ }^{52} e t$, avec lui, le grand jeu des semblances émanant de la divinité, le substantif semblance est resté en usage jusqu'au XVII ${ }^{\mathrm{e}}$ siècle, avec de multiples références encore possibles à l'apparence extérieure, à la ressemblance, à la représentation picturale. Toutes possibilités où il est déjà et depuis longtemps concurrencé par image, dont l'emploi dans la locution à l'image de, rare au Moyen Age, s'est considérablement développé par la suite, au XVII ${ }^{e}$ siècle notamment. Quant à la première vraisemblance ${ }^{53}$ à être signalée comme unité lexicale, elle se trouve dans un document d'archives du milieu du XIV siècle, où elle actualise la notion, sans doute assez nouvelle, de " vraisemblance " dans sa composante de « forte probabilité $~_{54}$.

Dans son évolution et la restructuration qui s'y opère des concepts par lesquels elle manifeste sa perception du monde, la langue française, en même temps qu'elle versait définitivement semblant du côté de la simulation ${ }^{55}$, a fini par substituer tout aussi définitivement image à semblance, mais en perdant ce qui est resté propre au lexème sembl - : l'évaluation par un récepteur de ce qui lui est donné à voir/à entendre et qui désormais ne peut jamais rendre compte de l'absolu de la vérité. 


\section{BIBLIOGRAPHIE}

\section{Principaux textes cités :}

Chrétien de Troyes, Le Conte du Graal, éd. F. Lecoy, Paris, Champion, 2 vol. CFMA, 1978.

L'Estoire del saint Graal, éd. J.-P. Ponceau, Paris, Champion, 2 vol. CFMA, 1997 (abrégé ESG).

Lancelot, roman en prose du XIIIe siècle, éd. A. Micha, Paris-Genève, Droz, 8 vol. TLF.

Merlin, roman du XIII' siècle, éd. A. Micha, Genève, Droz, 1979, TLF.

Perlesvaus, éd. W. A. Nitze et T. A. Jenkins, Chicago, The University of Chicago Press, 2 vol. , 1932.

Philippe de Thaon, Comput, ed. by I. Short, ANTS, Plain Texts Series 2, 1984.

La Queste del saint Graal, éd. A. Pauphilet, Paris, Champion, CFMA, 1921.

Robert de Boron, Joseph d'Arimathie, A Critical Edition of the Verse and Prose Versions by R. O'Gorman, Toronto, 1995, Pontifical Institute of Mediaeval Studies.

La Suite du Roman de Merlin, éd. G. Roussineau, 2 vol. , Genève, Droz, 1996, TLF.

Wace, Le Roman de Brut, éd. I.D.O. Arnold, 2 vol. , Paris, SATF, 1983-1940 ; La geste du roi Arthur, présentation, édition et traduction par E. Baumgartner et I. Short, Paris, 10/18, 1993.

\section{Choix d'études sur les textes du Graal :}

BAUMGARTNER, E. (1981) L'Arbre et le Pain. Essai sur la Queste del saint Graal, Paris : SEDES.

- «Robert de Boron et l'imaginaire du livre du Graal », repris dans De l'histoire de Troie au livre du Graal, Paradigme, Orléans, 1994, 487-496.

BAUMGARTNER, E. et ANDRIEUX-REIX, N. (2001) Le Merlin en prose. Fondations du récit arthurien, Etudes littéraires, Recto-verso, PUF.

COMBES, A. et BERTIN, A. (2001) Ecritures du Graal, Etudes littéraires, Recto-verso, Paris : PUF.

DUBOST, F. (1998) Le Conte du Graal et l'art de faire signe, Paris : Champion.

LEUPIN, A. (1982) Le Graal et la littérature, Paris : L'Age d'Homme, « Lettera ».

MELA, C. (1979) Blanchefleur et le saint homme ou la semblance des reliques, Paris : Ed. Seuil.

POIRION, D. (1994) «Semblance du Graal dans la Queste, repris dans Ecriture poétique et composition romanesque, Orléans, Paradigme, 201-215.

SEGUY, M. (2001) Les romans du Graal ou le signe imaginé, Paris : Champion.

STRUBEL, A. (1989) La Rose, Renart et le Graal, Paris : Champion.

SZKILNIK, M. (1991) L'Archipel du Graal, Genève : Droz.

VALETTE, J.-R. (1999) « Personnage, signe et transcendance dans les scènes du Graal (de Chrétien de Troyes à la Queste du saint Graal) dans Personne, personnage et transcendance (XII ${ }^{e}$-XIII ${ }^{e}$ siècles) sous la dir. de M.E. Bély et J.-R. Valette, Lyon, Presses Universitaires de Lyon, 1-24.

Il n'y a quasiment pas d'études linguistiques sur semblance ; à part de brèves allusions dans A. François, (1950) La désinence -ance dans le vocabulaire français, Genève-Lille, 70-71 et dans J. Renson, 
(1962) Les dénominations du visage en français et dans les autres langues romanes, Paris, 436 sq., on citera l'article de

VOICU, M. (1992) «"Semblance" et "senefiance" ou de la lecture des signes dans Le Conte du Graal ", dans Revue roumaine de linguistique, t. 37, 79-87.

\section{NOTES}

1. En semblance de notre ancienne, et celle-ci durable, amitié pour Michèle Perret, à qui nous dédions cette étude.

2. On sait néanmoins comment, à la fin du XIII ${ }^{\mathrm{e}}$ siècle, Jean de Meun introduit le personnage de Faux-Semblant pour qualifier une apparence entièrement composée, en désaccord avec la personnalité vraie de l'hypocrite, masquant ses ruses et ses vices sous son habit de frère mendiant.

3. Voir ci-après $§ 1.1$.

4. Sans être impossible, semblant est rare dans la forme en + substantif + de (étudiée plus loin, $\S$ 1.2.1.) ; on signalera toutefois : Saints Brendans vit un diable en semblant d'un enfant ethyopien (Arch. Doubs) ; ce est l'Anemis qui lor fait venir leur choses a profit en semblant de bon eür (Philippe de Novare, Quatre âges... 208) ; de même pour semblance rare dans les autres formes syntaxiques : trei poete.../ les asistrent par tel semblance / que sor chascuns ot tresgeté / une image de grant beauté (Troie 14670) ; mout font laide samblanche (Bâtard de Bouillon 5615); n'en fere semblance ne chiere (Dolopathos 192) [citations faites la première d'après le dictionnaire de Godefroy, les autres d'après celui de Tobler-Lommatzsch].

5. Voir ci-après note 8 .

6. Ex. et se vos me faites bele chiere il ne m'en osseront ja faire samblant (Merlin P 39. 58/59).

7. un semblant de est attesté à partir du $17^{\mathrm{e}}$ siècle.

8. Voir l'opposition entre dire sa semblance (ex. du Moniage Guillaume II 3030) et - ci-dessous - dire de son semblant.

9. Voir toutefois note 4.

10. Voir aussi plus haut (début du § 1.1.2.).

11. et non de simple forme substantive constituant d'une locution verbale ou adverbiale.

12. Pour l'exception représentée par la veraie semblance de la Queste voir $\S 3$.

13. On notera la persistance de cette double possibilité, inscrite dans l'histoire du lexème sembl-, avec le double rattachement étymologique du verbe sembler à la fois à similare et à simulare; similare (formé en bas-latin sur l'adjectif similis) signifiant « être pareil à » a dès lors concurrencé dans cette signification l'ancien simulare, lequel a toujours pu signifier aussi bien " être pareil à » que « simuler », «feindre ».

14. Semblance est la traduction française de similitudo: sulunc la semblance de serpent (secundum similitudinem sepentis) (Oxford Ps. 57.4).

15. La première occurrence répertoriée de similitude se trouve dans la Queste, p. 38, 1. 13.

16. De même pour la semblance des deux femmes et en semblance de... (ESG 562.5 cité § 1.2.2. et $\$ 4$ )

17. A distinguer de a la semblance de, laquelle entre dans les autres cas, cités $§ 1.2 .2$., de semblance pouvu d'un déterminant.

18. où la concurrence s'observe avec en guise de : voir fin du §1.2.2.

19. Voir le commentaire détaillé de cette occurrence ci-après $\S 2.1$.

20. La présence de ce récepteur est, on l'a vu, une caractéristique du lexème sembl- (voir début du $\S 1$.$) .$

21. Voir § de conclusion. 
22. On notera, chez le même auteur, les emplois comparables de figure et de semblance: voir fin $\mathrm{du} \S 1.2 .2$.

23. Voir aussi ci-dessus ESG 548.10.

24. Ce qui ne met pas en jeu la chronologie de ces écritures : voir $§ 3$.

25. Voir plus loin $\S \S 3$ et 4.

26. Voir aussi, éd. cit., vv. 27-31.

27. Voir E. Baumgartner et N. Andrieux-Reix (2001).

28. Très différent en cela des diverses semblances de la beste de l'ESG, elles, concomitantes.

29. entièrement comparable à la droite semblance du même Merlin, citée précédemment.

30. Texte que l'on peut désormais comparer avec sa version en prose grâce à l'édition synoptique d'O'Gorman à laquelle nous empruntons toutes nos citations.

31. On notera cependant que le Joseph utilise aussi semblance pour désigner une apparence destinée à tromper. Ainsi, au v. 2121 des diverses semblances prises par les démons ou de la semblance trafiquée que se donne Moïse aux vv. 2702 et 2752.

32. On se reportera notamment aux vv. 1507-8, 1531-2, 1548, 1555, 1563, 1569, 1587, 1613,1667, 1672, 1696, 1727, 1746.

33. Egalement dénommé au v. 1590 suaire. Voir en complément la note d'o'Gorman à la ligne 630 du texte en prose.

34. Le nom de Verrine, donné à la femme dans le Joseph en vers peut aussi renvoyer à la "verrine", la verrière traversée par le rayon de soleil en «semblance» du mystère de l'Incarnation. Mais il s'agit alors d'une semblance, d'une comparaison et non de la trace même laissée par le Christ.

35. Dans son usage ordinaire, semblance est engagé dans une représentation, intégrant donc la possibilité d'une falsification, à tout le moins d'une inexactitude ; or, pur transfert physique de traits, extérieur donc à toute mimesis de fiction, la Véronique est une reproduction d'un type particulier parce que s'y abolit la possibilité d'un écart; cette particularité lui a valu un traitement lexical variable selon les textes, certains l'excluant des références opérées par semblance: ainsi l'ESG paraît comme refuser à la vraie icône le nom de semblance (alors qu'elle l'accorde à la desloiale; voir $\S 4$.), auquel elle préfère celui de figure ( $\$ 43,1.16$ et 19) ou d'empriente ( $\$ 44,1.13)$.

36. Voir les vv. 893-928.

37. On se reportera sur ce point aux analyses de F. Dubost (1998) et de M. Séguy (2001).

38. Voir E. Baumgartner (1994).

39. Rappelons que, contemporain sans doute du Joseph en vers de Robert, le Conte du Graal n'accueille qu'une semblance toute profane, celle de Blanchefleur, rappelée par trois gouttes de sang sur la neige, et qui fait cependant accéder pour la première fois Perceval le nice à la conscience du souvenir.

40. Ces différentes visites se situent au T. VI de l'éd. A. Micha du Lancelot. Elles sont étudiées en détail dans M. Séguy (2001).

41. Le terme est notamment utilisé p. 304, 1. 7226 pour désigner les cinq manieres sous lesquelles le Graal apparaît à Arthur eu secré de la messe (au moment de la consécration des espèces).

42. Voir par exemple l'apparition à Arthur du Crucifié (éd. cit., p. 36) du Graal en cinq muances (éd. cit., p. 304) ainsi que les apparitions réservées à Gauvain, p. 119.

43. Voir par exemple, p. 38, la similitude qu'établit l'ermite entre la venue du Christ et celle de Galaad, tout en précisant que la comparaison porte sur la (re)semblance, mais non sur la hautece, sur l'importance inégalable de la venue du Christ.

44. Mais le diable peut troubler le jeu en se présentant par exemple sous la semblance (transparente) d'une belle demoiselle...

45. Pour une position contraire, voir J.-P. Ponceau, L'Estoire du saint Graal, éd. cit., pp. XI-XIV.

46. Voir Michèle Szkilnik (1991). 
47. Sur cet épisode, voir M. Séguy, «Hippocrate victime des images. A propos d'un épisode déconcertant de l'ESG», Romania 2001 (119), pp. 440-464.

48. Sur cet épisode, voir C.-A. Van Coolput, «La poupée d'Evalac ou la conversion tardive du Roi Mordrain » dans Continuations. Essays on Medieval French Literature and Language in honor of John L. Grigsby, éd. N. Lacy et G. Torrini-Roblin, Birmingham, Alabama, Summa, 1989, pp. 163-172.

49. La « déloyauté » ainsi étiquetée n'est pas une infidélité d'image entre la copie et son modèle, mais une perversion apportée par l'usage qui en est fait.

50. La question de ces éventuelles relations a elle-même suscité de nombreuses discussions : voir A.Strubel (1989) et D. Poirion (1994).

51. Dans la Queste, depuis longtemps remarquée comme imprégnée de l'esprit de Cîteaux, le Graal se révèle l'équivalent du Deus absconditus de la mystique cistercienne, et la vraie semblance en serait comme la gnose : l'accès direct à la connaissance par la vision ; voir Guillaume de SaintThierry (Commentaire sur le Cantique des cantiques) traduit dans A. Michel, Théologiens et mystiques du Moyen âge, Gallimard, 1997, p.299.

52. Tous les textes postérieurs à la Queste se situent en effet, comme l'ESG, le Tristan en prose, la Suite du Merlin, etc. dans un temps antérieur à l'achèvement de la quête par Galaad

53. Il est oiseux, pour le français médiéval, de mettre ou non un blanc entre les deux composantes; on signalera toutefois que, dans l'histoire de l'orthographe, c'est avec un trait d'union/désunion que le vocable a été enregistré dans le Dictionnaire de l'Académie, où il ne sera consigné en un seul mot graphique qu'à partir de 1762.

54. Si les ennemis du royaume estoient si pres dela ville de Reims que ce fust presomption et vraisemblance qu'ils veinssent assaillir la ville (c. 1358, Traité d'alliance, Archives administratives de la ville de Reims, cité par Godefroy). De cette notion, le français garde quelques traces très sporadiques à partir du milieu du XIII ${ }^{\mathrm{e}}$ s., où elle commence à être actualisée dans d'autres formes du paradigme de sembl- et qui apparaissent comme des latinismes partiels : verisemblable presumption dans le Roman des Sept Sages, voirsemblant, voirsemblable chez Brunet Latin (toutes choses voiressemblables ne sont pas voires); mais ces formations apparaissent souvent comme des hapax avant le développement d'une véritable fréquence d'emploi observable pour vraisemblance, qui ne paraît pas remonter au-delà du XIX ${ }^{\mathrm{e}}$ siècle (cf. TLF).

55. Jusqu'au XVI ${ }^{\mathrm{e}}$ siècle inclus, faire semblant a pu signifier «donner toutes les apparences de », « manifester ", sans que cette extériorisation corresponde nécessairement à une feinte.

\section{AUTEURS}

\section{NELLY ANDRIEUX-REIX}

Université Paris 3 - Sorbonne Nouvelle

EMMANUÈLE BAUMGARTNER

Université Paris 3 - Sorbonne Nouvelle 